\title{
The Properties of Open Cluster Binaries Based on High-Resolution Imaging Surveys
}

\author{
Jenny Patience \\ IGPP, LLNL, 7000 East Ave. L-413, Livermore, CA 94550 \\ Gaspard Duchêne \\ Laboratoire d'Astrophysique, Observatoire de Grenoble, Université \\ Joseph Fourier, B. P. 53, 38041 Grenoble Cedex 9, France
}

\begin{abstract}
With known properties such as age, distance and stellar density, young clusters provide ideal samples for binary star studies. We summarize recent results from high-resolution multiplicity surveys of IC 348, $\alpha$ Per, the Pleiades, the Hyades, and Praesepe. The statistics of the resolved companions are used to address binary star formation and evolution in clusters. Over the ranges of ages, densities and stellar masses covered by the clusters, it is found that the binary fraction is correlated with stellar density rather than age, and that both the binary fraction and binary mass ratio distribution depend upon mass. The impact of companions on X-ray emission and stellar rotation is also discussed.
\end{abstract}

\section{Introduction}

Extensive observations have established that binary stars are very common among field solar-type stars and are $\sim 2$ times more prevalent among $\mathrm{T}$ Tauri stars in the closest star-forming regions (Duquennoy \& Mayor 1991; Ghez et al. 1993, 1997; Leinert et al. 1993; Simon et al. 1995). A number of possible solutions have been suggested to explain the difference in binary fractions: disruption of systems over time (Ghez et al. 1993), an environmental factor which affects the efficiency of forming binaries (Durisen \& Sterzik 1994), separation distributions that peak at different values (Brandner \& Köhler 1998), and a high proportion of young brown dwarfs undetectable in surveys of older stars. The first part of this study investigates two of these scenarios by taking advantage of the varying ages and stellar densities probed by the cluster samples.

The second aspect of this work combines the datasets from the different clusters in order to study the statistics of the resolved binaries. The observational trends such as the mass ratio distribution and its dependence upon mass and separation provide tests of both capture (McDonald \& Clarke 1995, Sterzik \& Durisen 1998) and fragmentation (Clarke 1996, Bate \& Bonell 1997, Bate 2000 and this volume) binary star formation scenarios. Subsets of the cluster sample are also used to address two effects of companions on the primary $\mathrm{X}$-ray detections and rotation rates. 


\section{Detection Limits and Observed Samples}

\subsection{Observational Techniques}

Part of the cluster sample (faint $\alpha$ Per stars) and one of the comparison samples (Trapezium) were obtained with the Hubble Space Telescope, but the bulk of the data collected for this review comes from ground-based surveys. Two major techniques are currently used to recover the diffraction limit of ground-based telescopes: adaptive optics (AO) and speckle interferometry. Both methods are limited to relatively bright targets, either to satisfy the requirement of AO guiding or to ensure enough flux in the $\sim 0.1$ s individual speckle images. A key difference is that the star must be bright at the observed wavelength for speckle interferometry, but bright in the optical for $\mathrm{AO}$ imaging. The surveys conducted with speckle techniques were performed at $K$, while the shorter overheads associated with $\mathrm{AO}$ allowed a full coverage of the $J H K$ range.

Comparisons of surveys performed with both techniques must consider the significantly different detection limits. Speckle interferometry provides a nearly uniform detection limit down to the diffraction limit of the telescope, while AO imaging has a detection limit strongly dependent on the separation. For separations up to 2-3 times the diffraction limit, speckle is more sensitive, but AO is significantly more sensitive at larger separations, especially outside the seeing halo. In summary, speckle interferometry allows the detection of closer binaries, while $\mathrm{AO}$ is more suitable to detect very faint companions. In the first part of this review, all AO surveys were corrected for incompleteness, to compensate for their poor sensitivity at small separations.

\subsection{Samples}

In order to avoid selection biases, the definition of the samples must be strict. First, the targets must be confirmed members of the clusters, which is usually ensured through the use of proper motion surveys for the ZAMS clusters, combined with spectroscopic and photometric information. Large catalogues of cluster members are available for these clusters, and targets have been selected on the basis of their brightness and their $(B-V)_{0}$ color, which is an indicator of their masses.

For the very young PMS clusters, the youth of the stars, hence their physical association to the star-forming region, is assessed either through their location in color-magnitude diagrams or by the combination of several spectroscopic signatures: a late spectral type (typically $\mathrm{K}$ or $\mathrm{M}$ ), the $\mathrm{H} \alpha$ line in emission, and the presence of the Lithium resonance absorption line. Table 1 summarizes the cluster samples considered in this review. The total number of targets is given, along with the spectral types of the stars involved in the surveys. Over 1000 stars were studied within six clusters, including about 200 multiple systems.

\section{Studies of the Individual Clusters}

In this section, we focus on low-mass stars, i.e. stars with spectral types later than G0. In each cluster, ten to forty companions were discovered, allowing accurate estimates of the frequency of binaries. In the following sections, these 


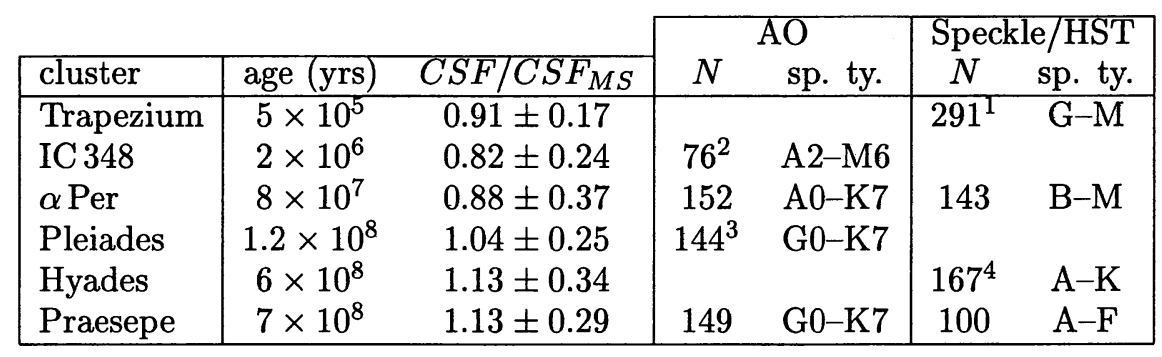

Table 1. Presented in the table are the age of the clusters, the binary fraction of their solar-type stars (normalized to the Main Sequence value), the total number and the full range of spectral types of the targets. References: ${ }^{1}$ Prosser et al. (1994); ${ }^{2}$ Duchêne et al. (1998); ${ }^{3}$ Bouvier et al. (1997); ${ }^{4}$ Patience et al. (1998).

clusters are compared with populations of low-mass stars in $\mathrm{T}$ associations, in order to study the evolution of the binary fraction and the dependence of the binary fraction on their physical properties.

\subsection{Binary Fraction as a Function of Age}

As can be seen from Figure 1(left), the binary fraction does not depend uniquely upon age. The behavior of the very young star-forming regions is quite complicated: some of the extremely young populations have a binary fraction in agreement with the Main Sequence population, while others display a visual binary excess up to a factor of the order of 1.6. Unlike the youngest populations, none of the observed ZAMS clusters has been found to host more binaries than the Main Sequence stars in the same orbital period range within Poisson error bars. The only possible exception is the 5-50 AU binaries in the Hyades.

It can be safely excluded that the binary fraction evolves with time in a universal fashion. In some cases, such as the Orion Trapezium or IC 348, any evolutionary decline is constrained to have occured within a million years or less. However, other similarly-aged populations appear to have kept most of their binaries. Therefore, if a mechanism responsible for a general decrease of the number of visual binaries does exist, it has to act on different timescales depending on the regions.

\subsection{Binary Fraction as a Function of Stellar Density}

The age is undoubtly a crucial parameter to describe a stellar population, but it may not be the most relevant in this context. Among the influential properties are: stellar density, gas temperature, and molecular cloud magnetic field strength. For the following comparison, the stellar densities of the various populations are roughly estimated from the number of known members within a radius of $0.5-1 \mathrm{pc}$; they range from a few tens to several thousands stars per $\mathrm{pc}^{3}$. In this scale, the ZAMS clusters are assumed to have been similar to the densest young cluster known in the solar neighborhood, the Trapezium cluster. 

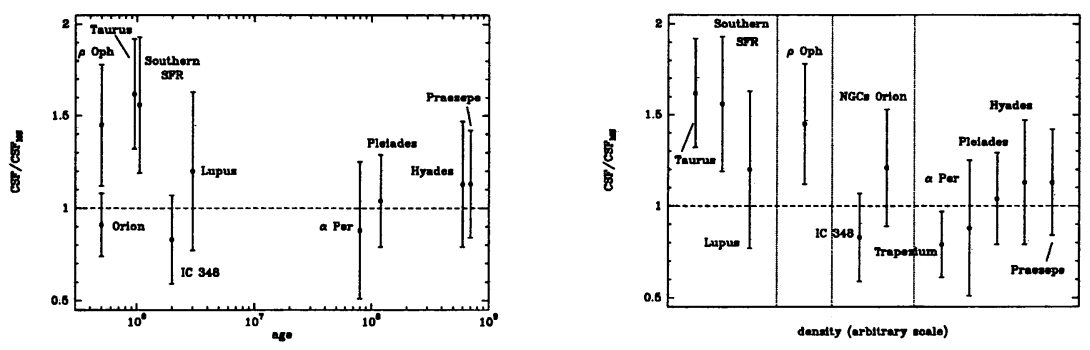

Figure 1. Dependence of the binary fraction on the age (left panel) and the density (right pannel) of the cluster. The vertical axis represents the binary fraction corrected for incompleteness for all mass ratios $q>0.1$ and divided by the Main Sequence value for the corresponding separation range.

Figure 1(right) suggests that a link does exist bewteen the binary fraction and the density, with a sharp dichotomy between clusters and loose associations. The former have binary fractions in agreement with the Main Sequence, and this behavior is noticeably independent on the age of the cluster. On the other hand, the associations of T Tauri stars systematically display significant excesses of visual binaries. Only the Lupus association shows a different behavior. It is clear, however, that the star formation process in a loose association leads to a very high binary fraction and that the number of binaries is not dramatically reduced during the first million years in these regions. This empirical link between the proportion of visual binaries and the density of the studied population can be interpreted as a causal relation between these properties, but it may also result from another property which would simultaneously affect both the stellar density and the binary fraction.

\subsection{Dynamical Evolution of Young Clusters}

The behavior described above is in qualitative agreement with the predictions of numerical simulations which follow the dynamical evolution of dense clusters over several thousands million years (e.g. Kroupa 1995). The initial condition for these simulations is a binary fraction on the order of $100 \%$. During the first phases of the cluster evolution, close encounters between binaries disrupt some of these sytems.

If the cluster is dense enough, the binary fraction can be reduced to the Main Sequence level within a million years or less, in agreement with the observations of the Trapezium cluster (Kroupa et al. 1999). On the other hand, the density of the $\mathrm{T}$ associations is so low that the number of encounters is extremely low (Clarke \& Pringle 1991), impeding the disruption of the binaries and accounting for the enhanced proportion of binary systems in these regions.

This qualitative agreement is further reinforced by the deficit of large visual binaries in clusters (Scally et al. 1999; Ghez, this volume; Macintosh et al. 2000), since these binaries are the most likely to be disrupted given their small binding 
energy. It is unclear, however, whether this mechanism can be fast enough in clusters significantly less dense than the Trapezium, such as IC 348, while remaining inefficient in the densest $\mathrm{T}$ association, $\rho \mathrm{Oph}$, which is intermediate between the Taurus-like regions and the bona fide clusters. Another puzzling issue is the mass-ratio distribution: numerical simulations predict that the lower the mass ratio the higher the probability for the binary to be disrupted. One could thus expect the mass ratio distribution in $\mathrm{T}$ associations to contain more binaries with $q<0.3$, which is not observed: all populations display a rather flat mass ratio distribution over the whole range $q=0.1-1$ (see Duquennoy \& Mayor 1991 for the Main Sequence, White [this volume] for T Tauri stars and below for the ZAMS clusters).

If the binary fraction is indeed driven by the close encounters in clusters, one can expect that more massive stars will retain more companions because of their deeper potential well, which prevents efficiently against binary disruption. Noticeably, OB stars in the Trapezium cluster (Preibisch, this volume) and in NGC 6611 (Duchêne 2000) display such a behavior, though estimates of the binary fraction for these stars strongly depend on incompleteness corrections. Furthermore, isolated Herbig AeBe stars also revealed much more companions than G dwarfs (Bouvier \& Corporon, this volume). On the other hand, intermediate mass stars in the various open clusters discussed here have a slightly lower binary frequency (see below). This contradictory result may result from the limited range of mass ratios probed by the observations, since a $3-4 M_{\odot}$ can host many very low-mass companions, which are extremely hard to detect around stars older than a few million years.

\section{Studies of the Combined Cluster Sample}

\subsection{Observational Properties}

Binary star properties provide observational tests of several binary formation models. Trends in the mass ratio $\left(q=m_{\text {sec }} / m_{\text {prim }}\right)$ distribution and the companion star fraction $[C S F=($ binaries $+2 *$ triples $) /($ singles + binaries + triples $)]$ are particularly important. Mass ratios are determined from the magnitudes of the individual components and mass-magnitude relations (Henry \& McCarthy 1993 for the lower mass stars and the appendix of Patience et al. 1998 for the higher mass stars). The sample for this aspect is limited to Main Sequence stars since the mass-magnitude conversion is more direct.

From the sample of 545 open cluster members with spectral types from B to $\mathrm{M}$ and with separations from 5-50 AU (for the Hyades) or 25-561 AU (for $\alpha$ Persei, the Pleiades, and Praesepe) several trends in the binary properties are evident(Patience et al. 2000). Considering binaries with $\Delta K \leq 4.0$, the $C S F$ decreases for increasing mass (equivalently decreasing color) as shown in Figure 2(left). As mentioned previously, the AO results on Herbig Ae/Be stars show a different trend, but are corrected for incompletness, while the speckle results are not. For the investigation of the mass ratio distribution, a cutoff of $q=0.4$ is imposed on the data to avoid incompleteness corrections. Combining the entire cluster dataset, the $q$ distribution given in Figure 2(right) increases slightly toward smaller mass ratios over the range of $q=0.4$ to 1.0 . The mass ratio distribution has a different shape, however, depending upon the primary 

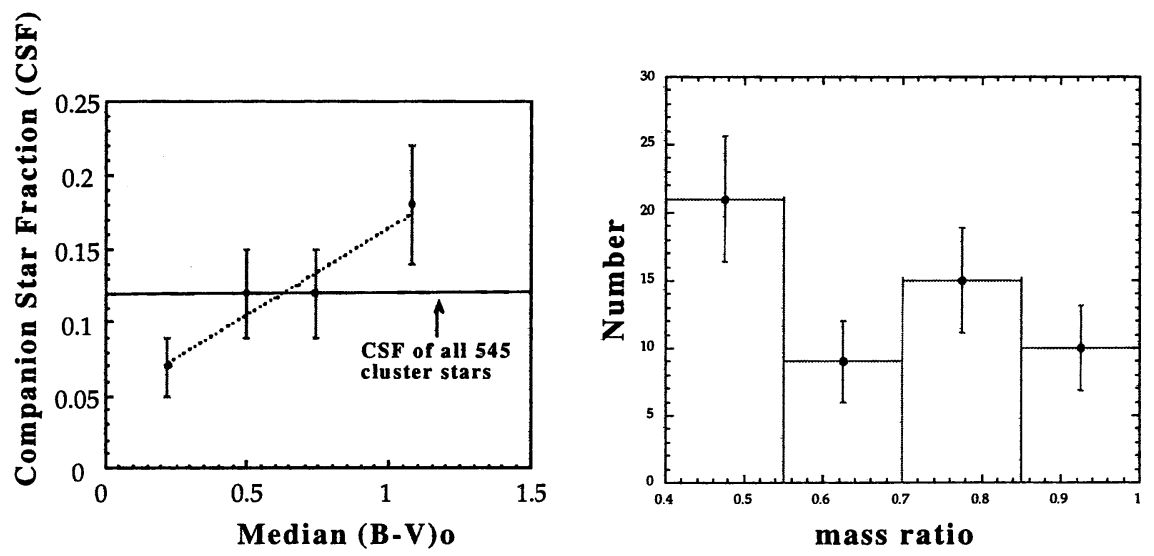

Figure 2. The left panel plots the companion star fraction $(C S F)$ as a function of color for cluster binaries with a $\Delta K=4.0$ cutoff; no corrections are applied. The $C S F$ is lower for bluer, higher mass stars. The mass ratio $(q)$ distribution over the range 0.4-1.0, displayed on the right panel, increases slightly toward lower $q$ systems.
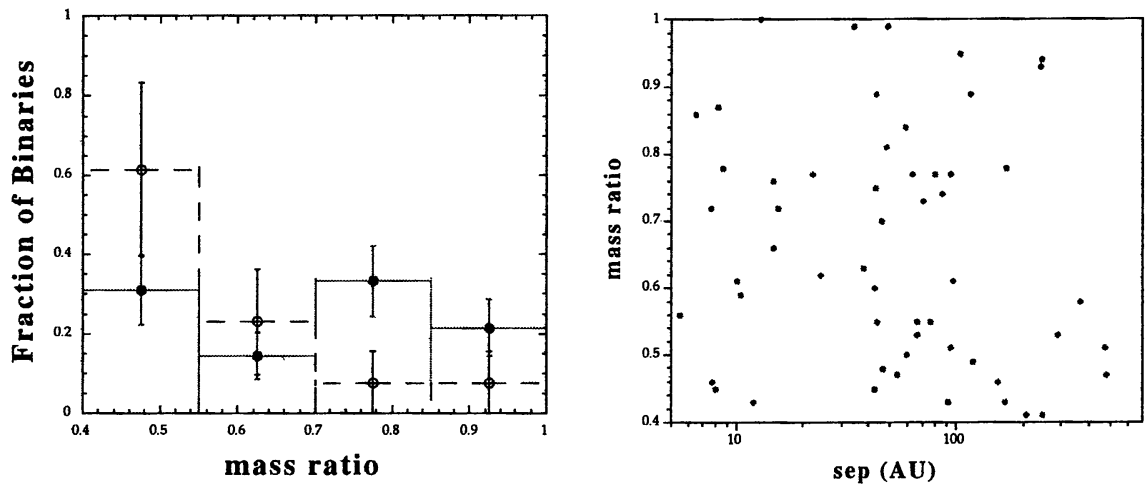

Figure 3. The dependence of the mass ratios on mass (left panel) and separation (right panel) are shown. The high mass (dashed) $q$ histogram is significantly different from the lower mass (solid) distribution; the dividing line is a spectral type of F7. Unlike the binary mass, the binary separation does not affect the mass ratio over the 5-561 AU range considered. 
mass; the $q$ distribution for higher mass primaries exhibits a steeper rise toward smaller $q$ systems as shown in Figure 3(left). Finally, Figure 3(right) plots the binary mass ratios as a function of separation over the 5-561 AU range covered by the cluster surveys - there is no correlation with separation.

\subsection{Comparisons with Binary Star Formation Models}

Capture in small clusters suggests that binaries form among the most massive pair of stars (Mc Donald \& Clarke 1995), but the observations show more lower mass binaries (Figure 2left) and more low mass ratios (Figure 2right). Including the effects of disks serves to randomize the companion masses and to allow a larger range of mass ratios (Mc Donald \& Clarke 1995) which is more consistent with the data. Another model of formation in small systems of stars (Sterzik \& Durisen 1998) predicts that more massive stars should have a higher proportion of binaries and a steeper slope to their mass ratio distribution. It is difficult to compare the observed $C S F$ vs. mass (Figure 2left) since the data are not sensitive to all possible companions, but the observed trend in mass ratio distribution (Figure 3left) matches the predicted effect. Both disk-assisted capture and dynamical decay of few-body systems make predictions that match the data.

Predictions from several fragmentation models can also be tested with the data. For samples such as the cluster data with a mass ratio sensitivity limit, fragmentation that is scale-free produces binaries with properties independent of mass (Clarke 1996); consequently, the observed mass dependence of both the $C S F$ (Figure 2left) and $q$ distribution (Figure 3left) are inconsistent with this model. Accretion simulations predict that systems with similar mass components should occur in closer separation or higher mass binaries (Bate \& Bonell 1997, Bate 2000). The limited number of $q \sim 1$ systems do not appear to vary with mass or separation. Another model with difficulties matching the observations is disk fragmentation which should preferentially form close and small mass ratio binaries. The occurence of similar component mass systems, and the absence of separation dependence on the $q$ distribution suggest that this mechanism is not the most frequent. Accretion simulations modified to represent a clustered rather than loose environment are more successful in reproducing the observational trends - more low $q$ systems among the massive stars (Bate $2001 \mathrm{~b}$ ), as is seen in the data. Fragmentation which is scale-dependent due to factors such as magnetic fields may also result in binaries with properties more similar to those observed.

\subsection{Effects of Companions on Stellar Properties}

Binaries and X-ray Emission from $A$ stars: Companion stars may be an important factor in explaining the X-ray detections from late-B and A type stars. Although X-ray emission is observed from stars located throughout most of the H-R diagram, B6 to A5 stars lack both the strong winds of higher mass stars and the dynamo of lower mass stars believed to generate $\mathrm{X}$-rays along the rest of the Main Sequence (c.f. Pallavicini 1989). A possible explanation involves unresolved companions. The ROSAT X-ray satellite error boxes - typically $\sim 10^{\prime \prime}$ - may include later-type companions which could produce X-rays.

The sample for this part of the discussion is composed of the late- $\mathrm{B}$ and A stars $\left[-0.13<(B-V)_{o}<0.30\right]$ in $\alpha$ Persei, Praesepe, and the Hyades. 


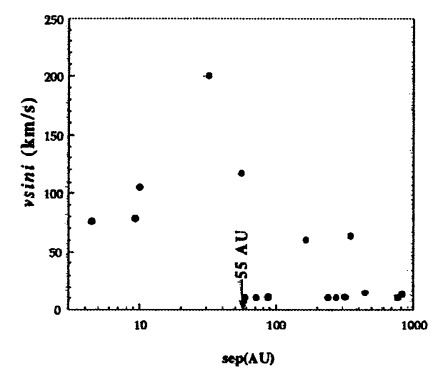

Figure 4. The rotational velocity is plotted as a function of binary separation for the solar-type stars in $\alpha$ Persei. Although the sample is limited, systems separated by less than $55 \mathrm{AU}$ have significantly higher vsini values.

These clusters have been targeted by both ROSAT and high resolution speckle multiplicity surveys; half of these stars have also been included in spectroscopic multiplicity surveys (e.g. Burkhart \& Coupry 1998, Abt \& Wilmarth 1999). The $C S F$ of the subset with X-ray detections is $C S F_{X-r a y}=0.6 \pm 0.2$ and the corresponding value of the subset without $X$-ray detections is a factor of 3 lower $C S F_{\text {noX-ray }}=0.21 \pm 0.06$, suggesting that companions may be responsible for the X-ray detections. A study of the younger counterparts to the cluster B and A stars - the Herbig Ae/Be stars - also revealed a larger number of binaries among stars having X-ray detections than among stars lacking X-ray emission (Zinnecker \& Preibisch 1994).

Binaries and Stellar Rotation: Since the solar-type stars in the youngest cluster $\alpha$ Persei are in a unique stage of stellar rotational evolution characterized by a wide range of rotational velocities (e.g. Prosser 1992.), the sample for the following analysis is limited to the youngest cluster $\alpha$ Persei. Two theories with opposing predictions suggest that binary stars may explain the range of observed rotational velocities. Both scenarios are predicated upon the assumption that stellar rotation is regulated by a star-disk interaction (Königl 1991, Armitage \& Clarke 1996) in which the stellar magnetic field is linked to the slowly rotating outer disk, providing a breaking force. One model suggests that binary stars disrupt (Bouvier et al. 1997), while an alternate theory suggests that binary stars should enhance (Armitage \& Clarke 1996) this breaking mechanism.

Among the 71 solar-type stars (F7 and later $\left.(B-V)_{o}>0.50\right)$ in $\alpha$ Persei, the measured vsini values cover the entire measurable range from $200 \mathrm{~km} / \mathrm{s}$ to $10 \mathrm{~km} / \mathrm{s}$ and extend beyond these limits. Regardless of the dividing $v \operatorname{sini} i$ value, the $C S F$ is not significantly different between the slow and rapid rotators. A similar result was obtained with an AO survey of the Pleiades (Bouvier et al. 1997). Although the presence of a binary companion is not directly correlated with the stellar rotation rate, the data do show a trend of increased rotational velocity for smaller separations. Figure 4 plots the set of all 17 solar-type binaries detected in $\alpha$ Persei. Dividing the sample of binaries at 55 AU results in a K-S test probability of only $0.2 \%$ that the close and wide systems have the same 
vsini distribution. The median vsini value is $105 \pm 51 \mathrm{~km} / \mathrm{s}$ for the binaries with separations $<55 \mathrm{AU}$, but only $11 \pm 20 \mathrm{~km} / \mathrm{s}$ for the systems separated by $>55 \mathrm{AU}$. Assuming a connection between disk lifetime and rotation rate, the $\alpha$ Persei data suggests that binaries with separations closer than 55 AU have shorter-lived circumstellar disks. This result is consistent with the observation of reduced millimeter and submillimeter flux from $\mathrm{T}$ Tauri binaries with separations less than 50-100 AU compared to wider binaries in several star forming regions (e.g. Jensen et al. 1996).

\section{Summary}

Based on high resolution surveys of numerous young clusters, we have addressed several issues in binary star formation and evolution. The proportion of binaries in both open clusters and star-forming regions is correlated with density rather than age, suggesting that the discrepancy in binary fraction between the field and some star-forming regions is the result of an environmental effect. Observational properties of the resolved open cluster binaries include: a $q$ distribution that rises slightly to smaller values, a mass dependence in both the proportion of binaries and the $q$ distribution, and no correlation between separation and mass ratio; these trends are most consistent with two forms of formation by capture. Among the cluster A stars, companions appear to explain the unexpected X-ray emission and among $\alpha$ Persei solar-type stars, the closer binaries $(<55 A U)$ exhibit more rapid rotation.

Continuing advances in telescopes and instrumentation will produce further discoveries in binary star studies; we conclude with only a partial list of topics for future investigations. With the large telescopes and interferometers under construction or being planned, it will be possible to resolve even closer binaries separated by only a few AU. The precision of planned astrometric missions should resolve many spectroscopic systems and allow accurate measurement of fundamental parameters like stellar mass. Longer wavelength wavefront sensors for AO systems will allow investigations of embedded clusters. Finally, coronagraphic techniques combined with adaptive optics should allow companion searches to extend well into the brown dwarf regime and even into planetary masses for younger or extremely nearby targets.

\section{References}

Abt, H. A., \& Wilmarth, D. W. 1999, ApJ, 521, 682

Armitage, P. J., \& Clarke, C. J. 1996, MNRAS, 280, 458

Bate, M. R., \& Bonell, I. A. 1997, MNRAS, 285, 33

Bate, M. R. 2000a, MNRAS, 314, 33

Bate, M. R. 2001b, IAU Symposium 200: this volume

Bouvier, J., Rigaut, F., \& Nadeau, D. 1997, A\&A, 323, 139

Bouvier, J., \& Corporon, P. 2001, IAU Symposium 200: this volume

Brandner, W., \& Köhler, R. 1998, ApJ, 499, L79

Burkhart, C., \& Coupry, M. F. 1998, A\&A, 338, 1073 
Clarke, C. J., \& Pringle, J. E. 1991, MNRAS, 249, 584

Clarke, C. J. 1996, MNRAS, 283, 353.

Duchêne, G., Bouvier, J., \& Simon, T. 1998, A\&A, 343, 831

Duchêne, G. 2000, Ph.D. thesis, University of Grenoble

Duquennoy, A., \& Mayor, M. 1991, A\&A, 248, 485

Durisen, R. H., \& Sterzik, M. F. 1994, A\&A, 286, 84

Ghez, A. M., Neugebauer, G., \& Matthews, K. 1993, AJ, 106, 2005

Ghez, A. M., McCarthy, D. W., Patience, J. L., \& Beck, T. L. 1997, ApJ, 481, 378

Ghez, A. M. 2001, IAU Symposium 200: this volume

Henry, T. J., \& McCarthy, D. W. 1993, AJ, 106, 773

Jensen, E. L., Mathieu, R. D., \& Fuller, G. A. 1996, ApJ, 458, 312

Königl, A. 1991, ApJ, 370, L39

Kroupa, P. 1995, MNRAS, 277, 1491

Kroupa, P., Petr, M. G., \& McCaughrean, M. J. 1999, New A, 4, 495

Leinert, Ch., Zinnecker, H., Weitzel, N., Christou, J., Ridgway, S. T., Jameson, R., Haas, M., \& Lenzen, R. 1993, A\&A, 278, 129

Macintosh, B., Zuckerman, B., Becklin, E., McLean, I. 2000, ApJ, submitted

McDonald, J. M., \& Clarke, C. J. 1995, MNRAS, 275, 671

Pallavicini, R. 1989, ARA\&A, 1, 177

Patience, J., Ghez, A. M., Reid, I. N., Weinberger, A. J., \& Matthews, K. 1998, AJ, 115, 1972

Patience, J., Ghez, A. M., \& Reid, I. N. 2000, in preparation

Prosser, C. F. 1992, AJ, 103, 488

Prosser, C. F., Stauffer, J. R., Hartmann, L., Soderblom, D. R., Jones, B. F., Werner, M. W., \& McCaughrean, M. J. 1994, ApJ, 421, 517

Scaly, A., Clarke, C. J., \& McCaughrean, M. J. 1999, MNRAS, 306, 253

Simon, M., Ghez, A. M., Leinert, Ch., Cassar, L., Chen, W. P., Howell, R. R., Jameson, R. F., Matthews, K., Neugebauer, G., \& Richichi, A. 1995, ApJ, 443, 625

Sterzik, M. F., \& Durisen, R. H. 1998, A\&A, 337, 113

White, R. J., \& Ghez, A. M. 2001, IAU Symposium 200: this volume

Zinnecker, H., \& Preibisch, Th. 1994, A\&A, 292, 152 